Psychotherapie: Praxis 
Die Reihe Psychotherapie: Praxis unterstützt Sie in Ihrer täglichen Arbeit - praxisorientiert, gut lesbar, mit klarem Konzept und auf dem neuesten wissenschaftlichen Stand. 
Inge Seiffge-Krenke

\section{Väter, Männer und kindliche Entwicklung}

Ein Lehrbuch für Psychotherapie und Beratung

Mit 27 Abbildungen und 3 Tabellen

Springer 
Inge Seiffge-Krenke

Mainz-Kostheim

Deutschland

ISBN 978-3-662-47994-0

ISBN 978-3-662-47995-7 (eBook)

DOI 10.1007/978-3-662-47995-7

Die Deutsche Nationalbibliothek verzeichnet diese Publikation in der Deutschen Nationalbibliografie; detaillierte bibliografische Daten sind im Internet über $>$ http://dnb.d-nb.de abrufbar.

(c) Springer-Verlag Berlin Heidelberg 2016

Das Werk einschließlich aller seiner Teile ist urheberrechtlich geschützt. Jede Verwertung, die nicht ausdrücklich vom Urheberrechtsgesetz zugelassen ist, bedarf der vorherigen Zustimmung des Verlags. Das gilt insbesondere für Vervielfältigungen, Bearbeitungen, Übersetzungen, Mikroverfilmungen und die Einspeicherung und Verarbeitung in elektronischen Systemen.

Die Wiedergabe von Gebrauchsnamen, Handelsnamen, Warenbezeichnungen usw. in diesem Werk berechtigt auch ohne besondere Kennzeichnung nicht zu der Annahme, dass solche Namen im Sinne der Warenzeichen- und Markenschutz-Gesetzgebung als frei zu betrachten wären und daher von jedermann benutzt werden dürften.

Der Verlag, die Autoren und die Herausgeber gehen davon aus, dass die Angaben und Informationen in diesem Werk zum Zeitpunkt der Veröffentlichung vollständig und korrekt sind. Weder der Verlag noch die Autoren oder die Herausgeber übernehmen, ausdrücklich oder implizit, Gewähr für den Inhalt des Werkes, etwaige Fehler oder Äußerungen.

Umschlaggestaltung: deblik Berlin

Fotonachweis Umschlag: ( ) Tom Wang / fotolia.com

Satz: Crest Premedia Solutions (P) Ltd., Pune, India

Gedruckt auf säurefreiem und chlorfrei gebleichtem Papier

Springer-Verlag ist Teil der Fachverlagsgruppe Springer Science+Business Media

(www.springer.com) 


\section{Vorwort und Danksagungen}

Das Thema Väter hat mich seit den ersten Veröffentlichungen 1997 kontinuierlich über fast 20 Jahre beschäftigt. In meinen umfangreichen Längsschnittstudien haben wir Väter im Familienkontext seit dem 14. Lebensjahr bis zum 30. Lebensjahr ihrer Kinder untersucht. Da wurde bereits zu Beginn deutlich, dass Väter einfach anders mit ihren Kindern umgehen als Mütter. Weitere Studien an Scheidungsvätern und Vätern chronisch kranker Kinder kamen hinzu und erweiterten die Facette dessen, was ein Vater ist, wie er sich verhält, wie er von seinen Kindern erlebt wird. Es wurde schnell offenkundig, dass es verschiedene Vatertypen gibt und es nicht sinnvoll ist, von »dem Vater « zu sprechen, und dass vor allem der Lebenskontext des Vaters unbedingt für das Verständnis von Vatersein herangezogen werden muss. Später haben wir bei einer großen repräsentativen Stichprobe von 20- bis 30-Jährigen geschaut, was für sie das Thema Generativität, $d$. h. Vaterwerden, bedeutet, und waren auf ein Hinausschieben der Vaterschaft als Entwicklungsaufgabe gestoßen, die die meisten jungen Leute in ihrem zukünftigen Leben anstrebten. Im Zuge meiner Beschäftigung mit Identität nahm die Entwicklung der männlichen Identität, die Entwicklung von Jungen und erwachsenen Männern einen wichtigen Raum ein. Drei neue Bücher, jenes von Hans Hopf (2014) über die »Psychoanalyse des Jungen«, jenes von Egon Garstick (2014), »Junge Väter in seelischen Krisen«, und das Buch von Jürgen Grieser (2014) über »Triangulierung « waren eine große Bereicherung für mein Nachdenken über Väter.

Mein ganz besonderer Dank gilt meinem Mann Dirk und meinen Söhnen David und Moritz, die all die Jahre eine Quelle der Inspiration für mich waren: Unvergessen bleibt, wie David und Moritz im Rahmen meiner Antrittsvorlesung an der Universität Mainz 1997 zum Thema »Väter« den »Sag Du doch mal was«-Papa inszenierten. Von meinen Söhnen habe ich auch das Interesse an historischen Bezügen zur Vaterschaft. Wie auch schon bei früheren Büchern hat Moritz wieder die schönen Grafiken hergestellt und mir bei den Bildern sehr geholfen.

Ein solches umfangreiches Buch kann natürlich nicht ohne die Hilfe vieler kollegialer Freunde und im intensiven geistigen Austausch mit Studenten und Mitarbeitern entstehen. Allen voran möchte ich meinem israelischen Freund und Kollegen Shmuel Shulman, Bar Ilan Universität, danken, mit dem ich mein erstes Buch über Väter verfasste und der seither, ebenso wie ich, von der Thematik Väter begeistert ist. Meinen Bonner und Mainzer Projektmitarbeitern, insbesondere Frank Kollmar, Annette Boeger, Martina Tauber, Sina Nitzko, Christian Skaletz und Carlosh von Irmer danke ich für ihre Unterstützung bei der Datenerhebung und Auswertung. Zahlreiche meiner ehemaligen Studenten haben mit mir an dem Thema in Form von Doktorarbeiten, Diplomarbeiten bzw. BA-Arbeiten gearbeitet, so Fabian Escher in seiner Diplomarbeiten über Vatertypen und seiner Doktorarbeit über Väter in Deutschland und Marokko und Marja-Lena Haid, die mit ihrer Doktorarbeit Einblick in das Leben von arbeitenden und arbeitslosen jungen Paaren mit und ohne Kinder gab. Die von mir betreuten Diplomarbeiten von Susanne Pfaff zu Vätern und erwachsenen Kindern bzw. von Tabea Schädel zu russischen und türkischen Vätern und ihren Kindern sowie die berührende Arbeit von Peter Flaig zum Tod des Vaters sind hier zu nennen, ebenso wie die BA-Arbeiten von Sandra Hamann, Franziska Pfeffer, Mara Marinovic, Marina Lind und Ives Steininger zu Fragen der Vaterabwesenheit, zu alleinerziehenden Vätern, Vätern in der 
Psychotherapie und zu psychisch kranken Vätern. Ihnen allen sei an dieser Stelle herzlich gedankt.

Es war mir immer sehr wichtig, neben meiner Forschungs- und Lehrtätigkeit auch »ein Bein in der Praxis« zu haben, und so blicke ich auf eine umfangreiche und bereichernde Erfahrung mit analytischen Kollegen und Kolleginnen zurück. Es ist mir daher ein besonderes Anliegen, Kollegen und Kolleginnen aus der Supervisionsarbeit zu danken wie Frau Nagel und ihrem Team von der Beratungsstelle Dreieich, Dr. Kohl und seinem Team, Kinderpsychosomatik, Darmstadt, sowie Christine Rother, Nicole Welter, Anne Grothe, Anuschka Leipnitz und Nadejda Schmidt. Bedanken möchte ich mich aber auch bei Monika Radecki vom Springer Verlag, die mich immer wieder zu diesem Buch ermutigt hat, immer für mich da war und viel Geduld und Einsatz bewiesen hat, als es an die Fertigstellung ging. Für die professionelle Begleitung des Buches danke ich auch Sigrid Janke (Projektmanagement) und Barbara Buchter (Lektorat).

Ich konnte mich glücklich schätzen, dass mich auf meinem Weg neben meinem eigenen Vater viele positive und kreative Vaterfiguren begleitet haben wie Albert Spitznagel, Zoltan Erdely, Gerd Heising, Horst-Eberhard Richter und Gerd Rudolf. Dafür bin ich besonders dankbar.

\section{Inge Seiffge-Krenke}

Mainz, den 9. Juni 2015 


\section{Inhaltsverzeichnis}

Inge Seiffge-Krenke

1.1 Theorien über Väter in der Psychoanalyse: Mythische Erhöhung und Schonung bei Freud

1.2 Der »vergessene Vater« in der Objektbeziehungstheorie, der Selbstpsychologie und der Bindungstheorie Anstiege in der Forschungsaktivität zu Vätern, aber methodische Defizite........ 5 Die Veränderung des Bildes vom Vater in der Forschung: Phasen der Vaterforschung. . 7

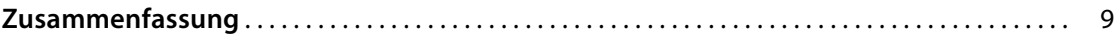

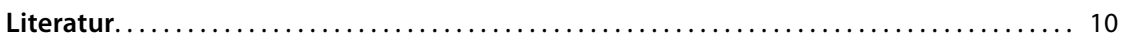

Die distinktive Bedeutung von Vätern in verschiedenen Entwicklungsphasen ihrer Kinder: Vom Babyalter bis zum jungen Erwachsenen

Inge Seiffge-Krenke

2.1 Väter von Säuglingen und Kleinkindern: Bindung,

Spielfeinfühligkeit und Triangulierung

Väter und Schulkinder: Lehrer, Herausforderer und Spielpartner

3.1 Der Sohn als »Spiegel des Vaters« und die selektive Liebe zum erstgeborenen Sohn. . 34

3.2 Die Disziplinierungs- und Lehrfunktion und die Bedeutung

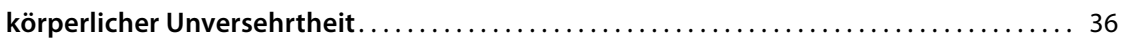

3.3 Liebevolle Vater-Sohn-Beziehungen und die Homophobie .................. 39

3.4 Aggressive und destruktive Tendenzen in der Vater-Sohn-Beziehung. . . . . . . . 40

$3.5 \quad$ Väter und Töchter: Verschiedenheit und Schutz $\ldots \ldots \ldots \ldots \ldots \ldots \ldots \ldots \ldots \ldots \ldots \ldots$

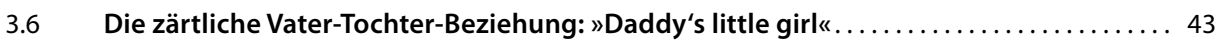

3.7 »Secure exitement«: Umgang mit körperlicher Reife und Sexualität der Tochter . . . . . 44

3.8 Die tüchtige Tochter und die Identifizierung mit dem Vater. . . . . . . . . . . . . . 47

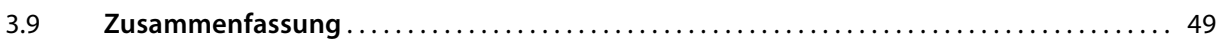

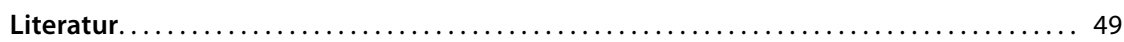

$4 \quad$ Vaterschaft im Wandel und verschiedene Vatertypen. 
Die Kosten der Veränderung von Vaterschaft:

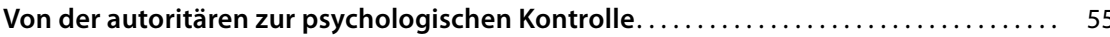
Väter und Mütter im Vergleich: „Co-parenting « und dennoch verschieden? ....... 76 Wird der Dritte zugelassen bzw. ein zu rascher Wechsel: Ist Papa heute eine Mama? .. 88

Literatur.

6.2 Vaterwerden verändert den Mann: Kinderwunsch, Unsterblichkeitsfantasien und die Veränderung des genealogischen Ranges....... 97 
7.6 Das Verhalten der Trennungsväter: Zwischen Rückzug,

„Disneyland daddy « und »Paternal banking« . .

7.7 Väterliches (selektives) Involvement als Puffer gegen

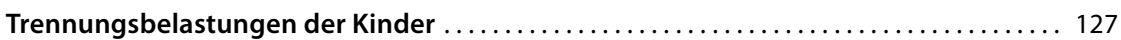

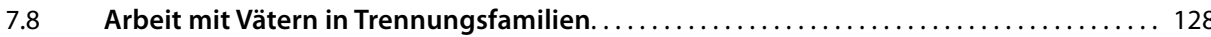

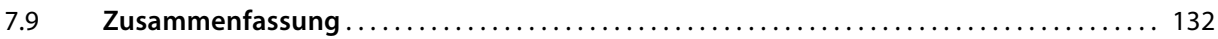

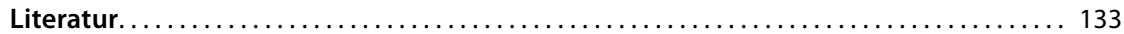

$8 \quad$ Alleinerziehende Väter - Gefährdung für die Gesundheit

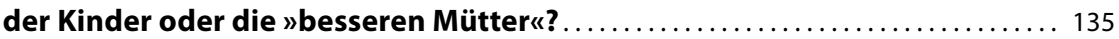

Inge Seiffge-Krenke

8.1 Alleinerziehende Väter in Deutschland: Häufigkeit, Ursachen,

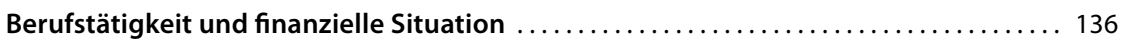

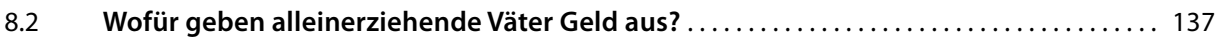

$8.3 \quad$ Gesundheit, Stress und Belastungen alleinerziehender Väter............... 138

8.4 Wie nehmen alleinerziehende Väter die Reaktionen ihrer

Umwelt auf ihre Rolle wahr?................................. 140

8.5 Gesundheitsversorgung, Auswirkungen auf das Wohlbefinden und die

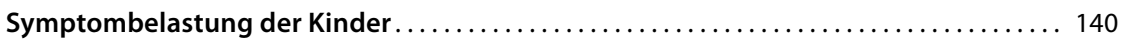

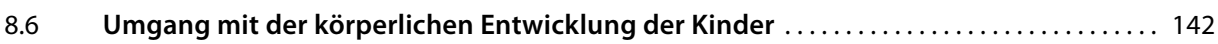

8.7 Andere Erziehungsverhaltensweisen beim alleinerziehenden Vater? . . . . . . . . . 142

$8.8 \quad$ Väterliches Involvement und Schulerfolg bzw. Schulabschluss der Kinder . . . . . . . . . 144

8.9 Familienkonzepte und die Bedeutung der ehemaligen Partnerin . . . . . . . . . . . . 145

$8.10 \quad$ Alleinerziehende Väter - die besseren Mütter? . . . . . . . . . . . . . . . . . . . 146

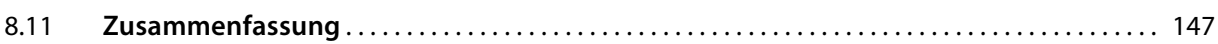

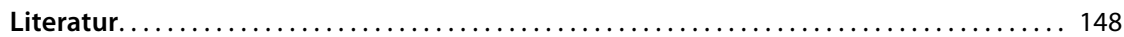

9 Der Tod des Vaters: Trauerarbeit und Erfahrungen mit dem Tod des Vaters ... 149 Inge Seiffge-Krenke

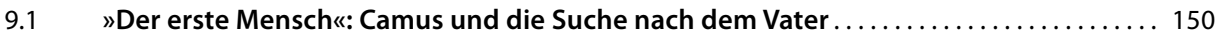

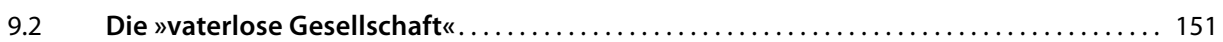

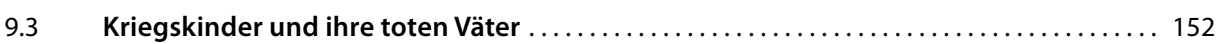

9.4 Einflussfaktoren und Verarbeitung des Vaterverlusts: Ein hilfreiches Modell für

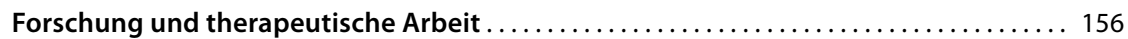

9.5 Faktoren, die die Verarbeitung des Tods des Vaters beeinflussen . . . . . . . . . . 157

9.6 Aktuelle Befunde zu den Auswirkungen des Tods des Vaters

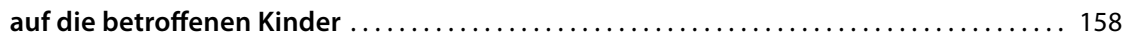

9.7 "... er muss rechtzeitig sterben«: Gibt es positive Folgen des Vaterverlusts? . . . . . 161

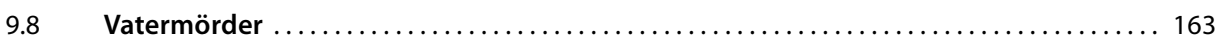

9.9 Die Verarbeitung des Todes des Vaters in der Psychotherapie: Eine Fallgeschichte... 164

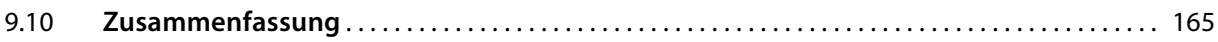

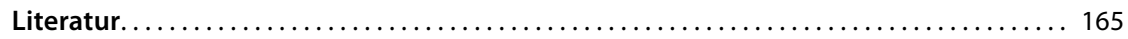

10 Kulturelle Einflüsse auf die Vaterschaft: Chance für eine

Veränderung oder Beharrung auf traditionellen Vaterkonzepten? ....... 167 Inge Seiffge-Krenke

10.1 Charakteristiken von Familien mit Migrationshintergrund. 
10.2 Unterschiedliche Werte in Familien: Individualismus versus Kollektivismus und die unterschiedliche Gewichtung von Verbundenheit und Autonomie

10.3 Vaterschaft im Einwanderungskontext: Defizit oder Resilienz? ................ 172

10.3.1 Einwanderungsväter als Musterbeispiel für Modernisierungsprozesse: Mehr Zeit für Kinder, väterliche Autorität auf dem Prüfstand . ...

10.3.2 Ähnliche Fokussierung auf das Erziehungsziel Autonomie bei deutschen, russischen und türkischen Vätern

10.3.3 Wandel der Rolle des Vaters in Marokko: Mehr Unterstützung

aber auch mehr Kontrolle, selbst bei erwachsenen Kindern ...

10.4 Faktoren, die zu mehr väterlicher Gewalt in Familien mit Zuwanderungsgeschichte beitragen

10.5 Väterliche Gewalt in Familien mit Zuwanderungsgeschichte

10.6 Auswirkungen von inkonsistentem väterlichen

Erziehungsverhalten und zu viel Kontrolle auf die Kinder

10.7 Väter aus anderen Kulturen in Beratungsarbeit und Psychotherapie............. 182

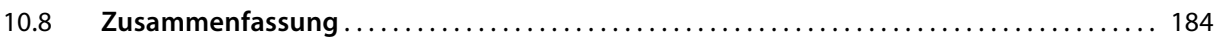

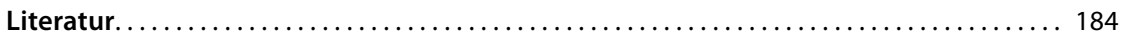

11 Väter und Psychopathologie: Risiko- oder Schutzfaktor? .............. 187

Inge Seiffge-Krenke

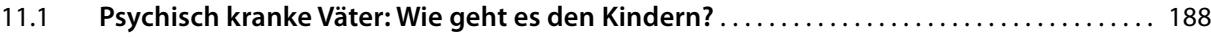

11.2 »Mother blaming?« oder: Welchen Beitrag leisten

Väter zur Psychopathologie ihrer Kinder? ............................. 191

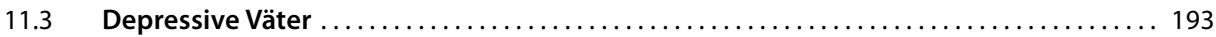

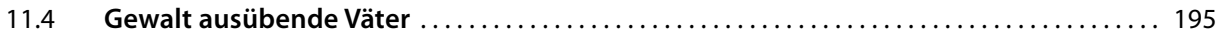

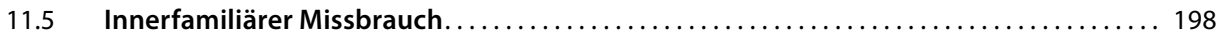

11.6 Väter als Ressource und Puffer bei familiären Belastungen . . . . . . . . . . . . . . . ... 200

11.7 Beratungsarbeit mit Vätern in verschiedenen Kontexten . . . . . . . . . . . . . . 202

11.7.1 Unterstützung väterlicher Funktionen: Vätergruppen und Vater-Kind-Wochenenden . . . . 203

11.7.2 Stärkung der väterlichen Kompetenz in der begleitenden Elternarbeit ........... 203

11.7.3 Arbeit mit Vätern in Familien mit chronisch kranken Kindern. . . . . . . . . . . . . . . . . . . . 204

11.7.4 Arbeit mit Vätern in Familien mit psychosomatisch erkrankten Kindern:

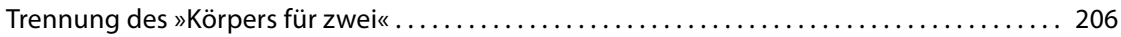

11.7.5 Beratungsarbeit mit Vätern in Familien mit entwicklungsbehinderten Kindern......... 208

11.7.6 Arbeit mit Vätern in Familien mit ADHS-Kindern und Kindern

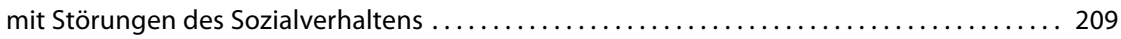

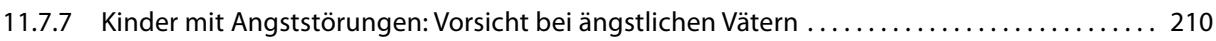

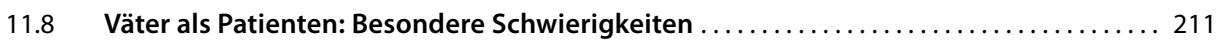

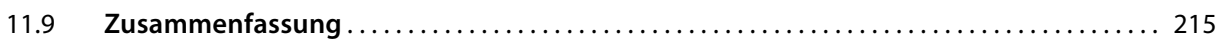

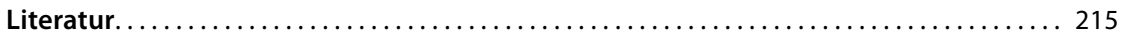

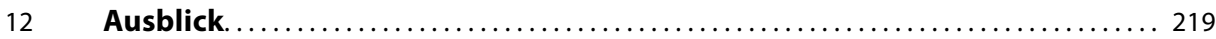

Inge Seiffge-Krenke

\section{Serviceteil}




\section{Die Autorin}

Prof. Dr. Inge Seiffge-Krenke hat Psychologie in Göttingen und Gießen studiert, 1981 promoviert und sich 1985 habilitiert. Nach Professuren in Gießen (für Medizinische Psychologie), Berlin und Bonn (Entwicklungspsychologie) war sie bis 2013 Leiterin der Abteilung Entwicklungspsychologie der Universität Mainz. Sie hat verschiedene Längsschnittstudien zur Familien- und Beziehungswicklung betreut. In einem von ihr geleiteten kulturvergleichenden Projekt wurde die Stressbewältigung bei Jugendlichen in 25 Ländern untersucht; gegenwärtig arbeitet sie mit Kolleginnen und Kollegen aus sieben Ländern zusammen, um den Zusammenhang zwischen (verzögerter) Identitätsentwicklung und Familienbeziehungen zu erforschen. Romantische Beziehungen, Vaterforschung, imaginäre Gefährten und die neu entdeckte Entwicklungsphase »emerging adulthood« sind weitere Schwerpunkte. Sie war Präsidentin der Europäischen Jugendforschung (EARA) und Gründungsmitglied der Organisation zur Erforschung des Emerging Adulthood (SSEA); in beiden Organisationen ist sie noch sehr aktiv. Sie ist seit 1983 Psychoanalytikerin (DPV/IPV) und seit 20 Jahren in der Ausbildung und Supervision von Ausbildungskandidaten für Kinder- und Jugendlichentherapeuten bzw. Erwachsenentherapeuten, niedergelassenen Therapeuten sowie in Kliniken und Beratungsstellen tätig. Seit 2005 ist sie im wissenschaftlichen Beirat der Lindauer Psychotherapiewochen und Sprecherin der Konfliktachse der OPD-KJ.

Für ihr Buch zur Behandlungstechnik bei Jugendlichen (»Psychoanalytische und tiefenpsychologisch fundierte Therapie mit Jugendlichen«) erhielt sie 2007 den Heigl-Preis. 